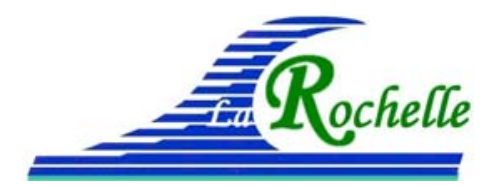

XVèmes Journées Nationales Génie Côtier - Génie Civil

La Rochelle, 29 au 31 mai 2018

DOI:10.5150/jngcgc.2018.008

(C) Editions Paralia CFL

disponible en ligne - http://www.paralia.fr - available online

\title{
Analyse de la courantologie dans le port de plaisance de La Rochelle
}

\section{Jean-Rémy HUGUET ${ }^{1}$, Isabelle BRENON ${ }^{1}$, Thibault COULOMBIER ${ }^{1}$}

1. LIENSs, Université de La Rochelle, 6 rue Olympe de Gouges, Bâtiment ILE, 17000 La Rochelle, France.

jean-remy.huguet@univ-Ir.fr ; isabelle.brenon@univ-Ir.fr

\begin{abstract}
:
Harbour siltation is a problem that exists as long as harbours exists and is intrisically linked to their primary function - provide shelter for anchorage and operative conditions for loading/unloading ships. In these semi-enclosed basins, characteristics of the flow are one of the main factors influencing the siltation and the water quality, since they govern the interaction between inside and outside waters and thus, determine the residence time of particles inside the basin. Les Minimes harbor, the main site of La Rochelle marina, and one of the largest recreational port of Europe, isn't spared by this problematic and has to face serious dredging operations major part of the year. In this context, a general study has been conducted in order to understand the 3D dynamic of sediment particles in connection with physical processes (wind surface stress, tidal forcing, baroclinic effects) and anthropic influence such as the frequent passage of vessels or structures like pontoons. From a methodological point of view, the project consists in an instrumental approach (ADCP, ADV, pressure and turbidity sensors, CTD, lagrangian buoys, ...) coupled with an operational model, TELEMAC 3D. The objective of this study is to determine the key driver of the fine sediment dynamics and to characterize the intra-harbor fluxes and exchange between open waters and the harbor. We will focus on the effect of floating docks on the hydrodynamics inside La Rochelle marina, comparing fluxes and residence times under several forcings (tide, meteo).
\end{abstract}

Keywords:

Numerical model, Currentology, Hydrodynamics.

\section{Introduction}

Les ports sont définis comme des endroits naturels ou artificiels permettant de protéger et/ou de stocker les bâtiments de navigation. Ils existent depuis aussi longtemps qu'existe le transport maritime. En plus d'une valeur économique indéniable, ils assurent au territoire auquel ils sont rattachés, une identité forte. (COLIN, l'identité des villes portuaires, 1992). Le port de plaisance de la Rochelle, fort de ses 5000 places, réparties sur plus de 15 hectares, est le plus grand de la façade atlantique européenne. Crée en 1972, il regroupe plusieurs sites dans cet ancien village de pêcheurs, devenu 


\section{Thème 1 - Hydrodynamique côtière}

une plateforme incontournable du commerce depuis le 12e siècle. Plus récemment, en 2014, le port a subi une extension sur son site principal, le port des Minimes, afin de faire face à une demande toujours plus forte de places à l'eau.

Les ports doivent aussi faire face au problème de l'envasement, comme toute région côtière protégée de l'action des houles et des courants. Il représente non seulement une entrave à la navigation mais aussi une nuisance environnementale via le piégeage de métaux lourds et de polluants chimiques. L'envasement d'un bassin portuaire dépend des conditions environnantes : les apports fluviaux, maritimes, les régimes de marée et de houle, la météorologie mais aussi de l'hydrodynamique interne associée à la configuration des bassins et des ouvrages maritimes. La ville de la Rochelle est bordée par des eaux côtières pouvant être très turbides du fait de la remise en suspension des sédiments au gré des marées, des coups de vent tempétueux mais aussi des crues. Son port de plaisance n'est pas épargné par l'envasement et doit dépenser près de $10 \%$ de son budget annuel à la réalisation des travaux de dragage, huit mois par an. Connaître la dynamique portuaire est donc un challenge dans cette zone où la sédimentation peut dépasser $50 \mathrm{~cm}$ par an dans certains bassins. Elle s'avère être complexe à modéliser : le trafic maritime et le positionnement des ouvrages tels que les pontons affectent ponctuellement à durablement la courantologie.

Ce travail présente les résultats d'une étude cofinancée par le port de plaisance de la Rochelle et de la région Nouvelle-Aquitaine, visant à mieux comprendre la dynamique sédimentaire dans le port des Minimes. Une approche instrumentale in situ couplée à une modélisation opérationnelle (TELEMAC 3D) nous permet de caractériser la circulation des masses d'eaux dans le port des Minimes sous l'action de la marée et de la météo.

\section{Description de la zone d'étude}

Le site d'étude principal, le port des Minimes est composé de 4 bassins comportant plus de 4500 places pour $15 \mathrm{~km}$ de pontons. Il est situé au fond de la partie nord du Pertuis d'Antioche, une zone maritime abritée par les îles de Ré et d'Oléron (figure 1). Il est connecté au nord au Pertuis Breton par un passage étroit entre le port Atlantique La Rochelle - La Pallice et l'île de Ré alors qu'à l'Ouest un large détroit entre les deux îles le connecte à l'océan. Sa partie sud rejoint le bassin de Marennes-Oléron caractérisé par la forte présence de bancs vaseux découvrants (POIRIER et al., 2010). L'ensemble de ces trois entités forme le pertuis charentais, une zone côtière relativement peu profonde, faisant la transition entre le continent et l'océan.

Le régime de marée est de type macro-tidal semi-diurne avec un marnage de moins de 2 mètres en morte-eaux, à plus de 6 mètres durant les vive-eaux. Les marées sont dominées par M2 dont l'amplitude peut atteindre par phénomène de résonance et de réfraction, plus de $1 \mathrm{~m} 80$ à l'intérieur des Pertuis (BERTIN et al., 2012). 


\section{XVèmes Journées Nationales Génie Côtier - Génie Civil \\ La Rochelle, 29 au 31 mai 2018}

Le climat de houle à l'année est caractérisé par des vagues d'Ouest à Nord-Ouest, d'une hauteur moyenne de $1.5 \mathrm{~m}$ et des périodes allant de 8 à $12 \mathrm{~s}$ (BERTIN et al., 2008). La houle peut être très énergétique à leur entrée mais diminue rapidement à mesure que l'on rentre dans les pertuis. Cependant, les vagues générées lors de gros coups de vents, combinées à de forts courants de marée, sont les principaux moteurs de la remise en suspensionsuspension de sédiments fins et contribuent à un niveau de turbidité élevé des eaux peu profondes des pertuis (LE HIR et al., 2010).

\section{Modélisation numérique}

Le modèle hydrodynamique TELEMAC-3D (EDF-DRD, 1997) permet d'étudier les écoulements à surface libre à partir des équations de Navier-Stokes. Dans cette étude, nous avons utilisé le mode non-hydrostatique de ce modèle afin de simuler la dynamique, de l'échelle régionale (Pertuis Charentais), à l'échelle locale (le port de plaisance de la Rochelle) sur une maillage non-structuré (figure 1).

L'espace géographique s'étend au large à plus de $35 \mathrm{~km}$ pour une longueur de près de $100 \mathrm{~km}$ du nord au sud. Il est discrétisé sur un maillage de 40099 nœuds dont la résolution varie de $2 \mathrm{~km}$ au large à près de 5 mètres dans le port des Minimes. Le modèle possède, sur la vertical, 8 niveaux sigma ce qui amène à plus de 320000 nœuds le maillage 3D. Il utilise un assemblage de différentes bathymétries du SHOM ainsi que d'autres plus récentes (Octobre 2017), effectuées au monofaisceau dans le port de plaisance et dans son chenal d'accès. La topographie des zones intertidales est fournie par le relevé LIDAR réalisé au lendemain de Xynthia dans le cadre du programme national Litto3D.

Le long de sa frontière ouverte, le modèle est forcé par 34 constituants tidaux $(\mathrm{O} 1, \mathrm{~K} 1$, P1, Q1, M2, S2, N2, K2, 2N2, MU2, NU2, L2, T2, M3, M4, MN4, MS4, M6, M8, EPS2, MSF, MSQM, MM, SSA, SA, S4, MKS2, MF, LA2, J1, N4, MTM, R2, S1) obtenus par interpolation linéaire à partir d'un modèle global de marée FES2014. Le forçage atmosphérique est réalisé sur tout le domaine à l'aide de données horaires CFSR (Climate Forecast System Reanalysis) fournies par le NCEP (National Center for Environmental Prediction).

Ces données horaires correspondent à la pression atmosphérique et au vent avec une résolution spatiale de $0.2^{\circ}$. Le pas de temps hydrodynamique est de $5 \mathrm{~s}$ et les simulations sont lancées sur 200 cœurs en parallèle, pour un wall-time de $40 \mathrm{H}$ si on veut simuler la circulation sur notre domaine pendant deux mois. 


\section{Thème 1 - Hydrodynamique côtière}

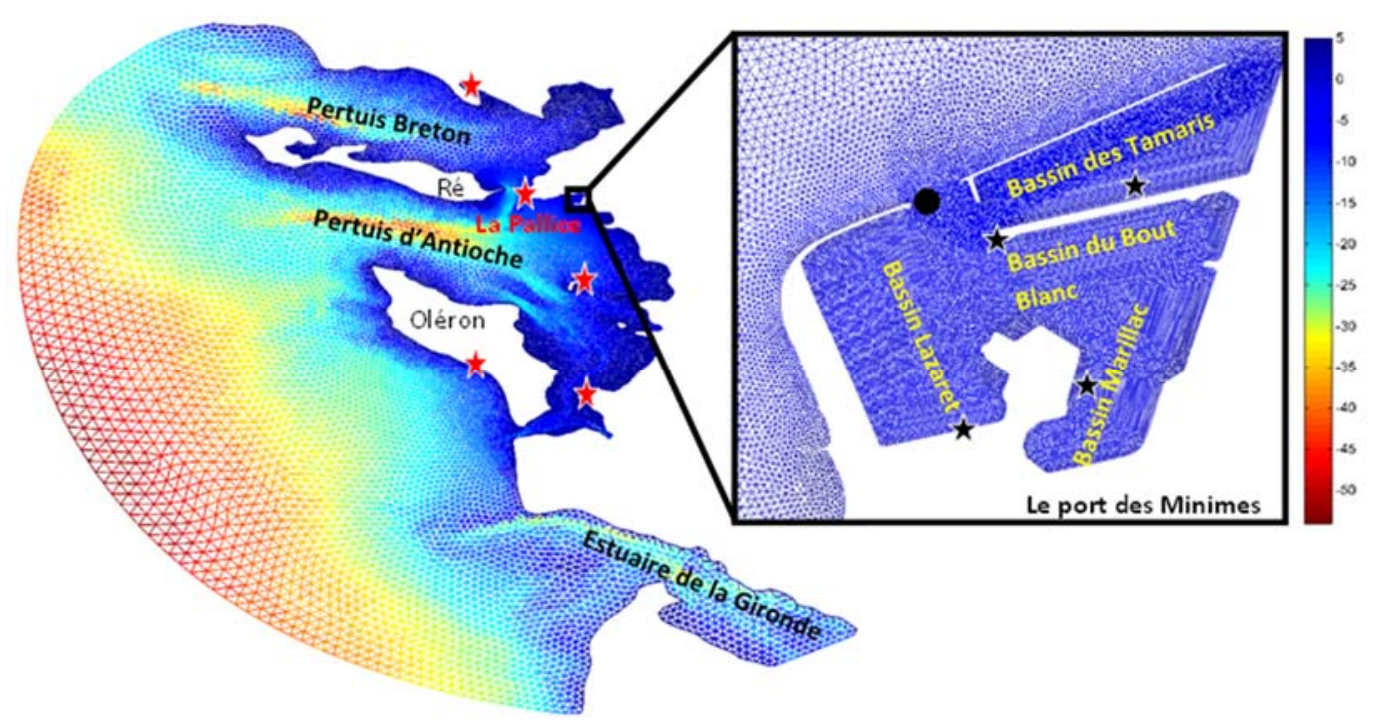

Figure 1. Extension du domaine de modélisation et visualisation du maillage non structuré utilisé pour l'étude (la résolution est de $2 \mathrm{~km}$ au large et $5 \mathrm{~m}$ dans la zone littorale au niveau du port des Minimes). Les entités géographiques les plus importantes sont nommées directement sur la figure. Les étoiles rouges correspondent aux marégraphes du réseau REFMAR (SHOM) - les étoiles noires correspondent aux capteurs de pression déployés par

l'Université de La Rochelle -le rond noir correspond au point d'ancrage de l'ADCP déployé par CREOCEAN en 2014 suite à l'extension du port.

\section{Résultats}

\subsection{Validation}

Les niveaux d'eau modélisés sont comparés aux niveaux d'eau mesurés par les différents marégraphes du SHOM dans le Pertuis Charentais (figure 1, étoiles rouges) ; mais aussi aux capteurs de pression déployés au sein des différents bassins par l'Université de la Rochelle début 2017 (figure 1, étoiles noires). Pour le port des Minimes, dans les différents bassins, le RMSE (Root Mean Square Error) est de 0.20 mètres avec un biais maximum d'environ 0.30 mètres alors que pour le port de la Pallice, le RMSE est de 0.24 mètres avec un biais maximum du même ordre. Les RMSE sont sensiblement les mêmes pour les différents marégraphes déployés dans tout le pertuis charentais.

Concernant la courantologie, les résultats sont comparés aux mesures ADCP réalisées par la cellule CREOCEAN en octobre 2014 après la fin des travaux d'extension dans le port. Le point de comparaison présenté en figure 3 est situé au niveau du chenal d'accès (figure 1, rond noir) où les vitesses de courant sont les plus fortes. En période de viveeaux, elles peuvent dépasser les $1.5 \mathrm{~m} / \mathrm{s}$ en début de flot et atteindre près de $0.8 \mathrm{~m} / \mathrm{s}$ en fin de jusant, lorsque l'espace alloué à l'écoulement est le plus petit. Le modèle reproduit bien ce comportement et reste en phase avec la réalité, avec néanmoins des 


\section{XVèmes Journées Nationales Génie Côtier - Génie Civil \\ La Rochelle, 29 au 31 mai 2018}

vitesses de jusant plus fortes que ce qui est observé. L'erreur maximum est de $10 \mathrm{~cm} / \mathrm{s}$ tandis que le RMSE est de $11 \mathrm{~cm} / \mathrm{s}$ pour les vitesses de surface et $6 \mathrm{~cm} / \mathrm{s}$ pour les vitesses de fond.
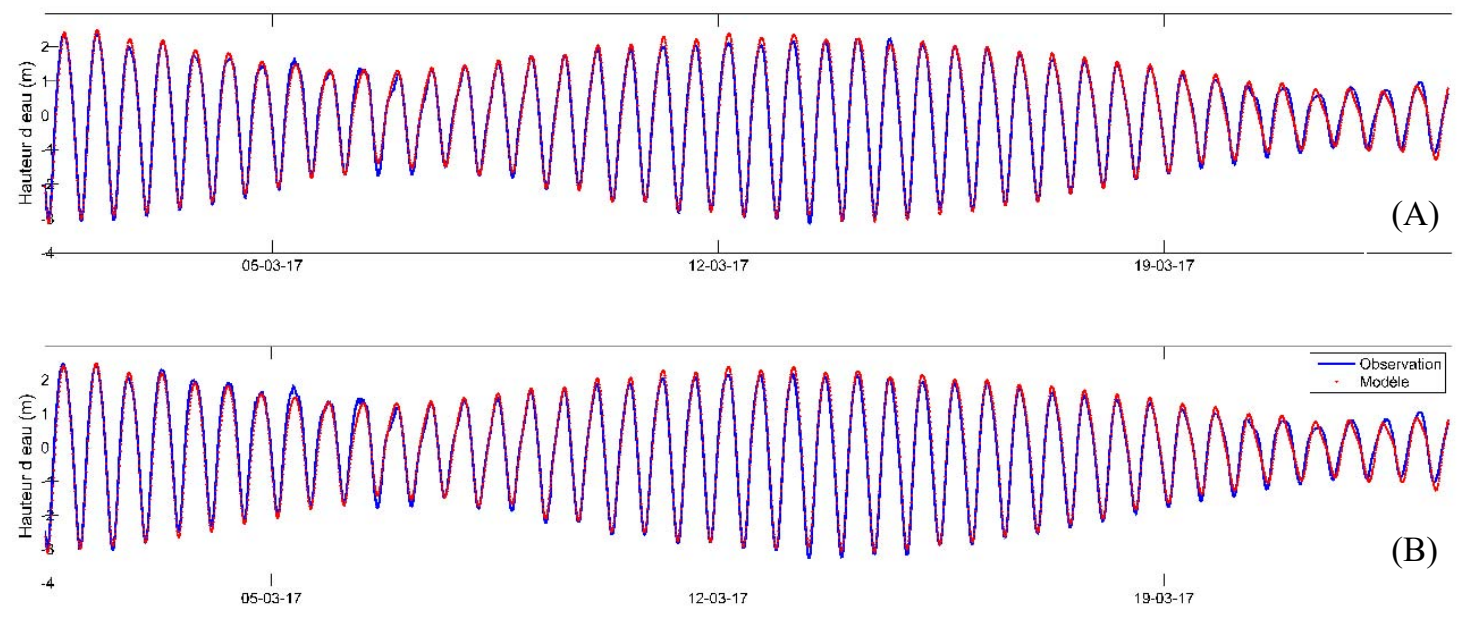

Figure 2. Comparaison des hauteurs d'eau modélisées et mesurées en mars 2017 à la station marégraphique de la Pallice (B) et au port des Minimes (A).
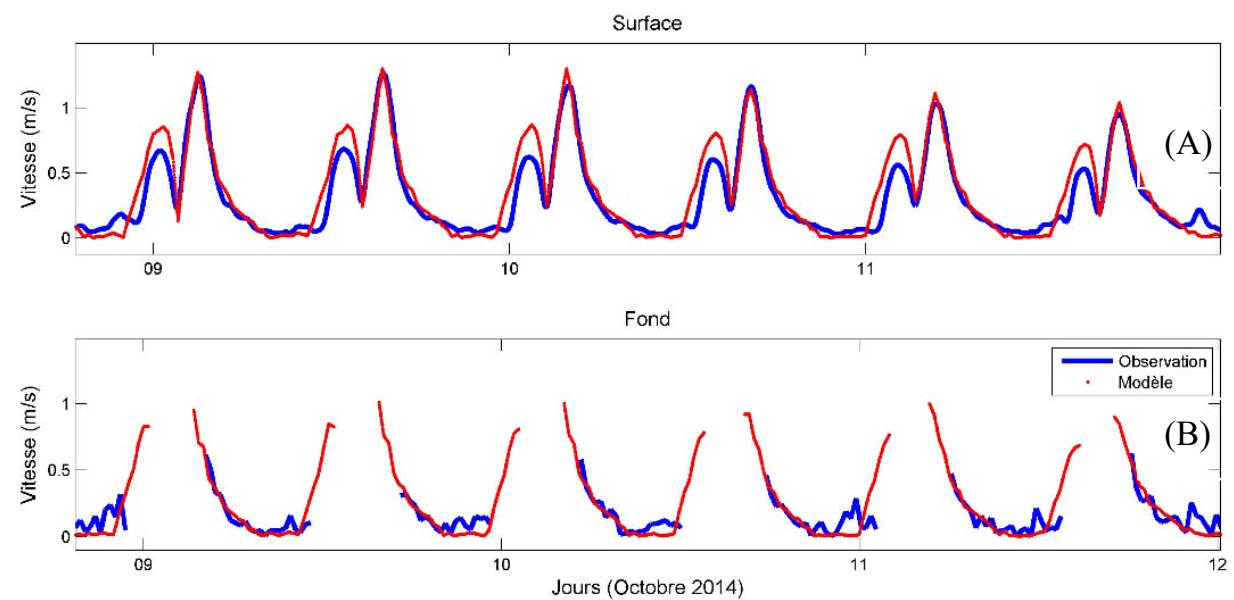

Figure 3. Comparaison des vitesses de courant observées et modélisées au niveau de l'entrée du chenal du port des Minimes (rond noir sur figure 1). En haut (A), les vitesses correspondent aux vitesses de surface alors qu'en bas (B), ce sont les vitesses de fond qui sont analysées.

\subsection{Circulation 3D différents régimes de marée}

L'étude courantologique a montré que les vitesses les plus élevées au sein du port des Minimes sont situées en fin de jusant et en début de flot. Ce comportement est analysé dans sa globalité avec l'action seule de la marée, en figure 4. Les résultats sont issus de simulations 3D pour une marée de vive-eaux (marnage $>6$ mètres) et sont moyennés 


\section{Thème 1 - Hydrodynamique côtière}

sur la verticale. Il apparaît clairement que les configurations courantologiques sont très différentes entre le flot et le jusant. Le flot est dominé rapidement, $2 \mathrm{H}$ après la basse mer, par la présence de tourbillons, ou gyres, à l'échelle des bassins. Ces tourbillons sont présents jusqu'à près de 2 heures après la pleine mer. Le jusant se caractérise, lui, par une courantologie faible dans les bassins et concentrée au niveau du chenal d'accès.
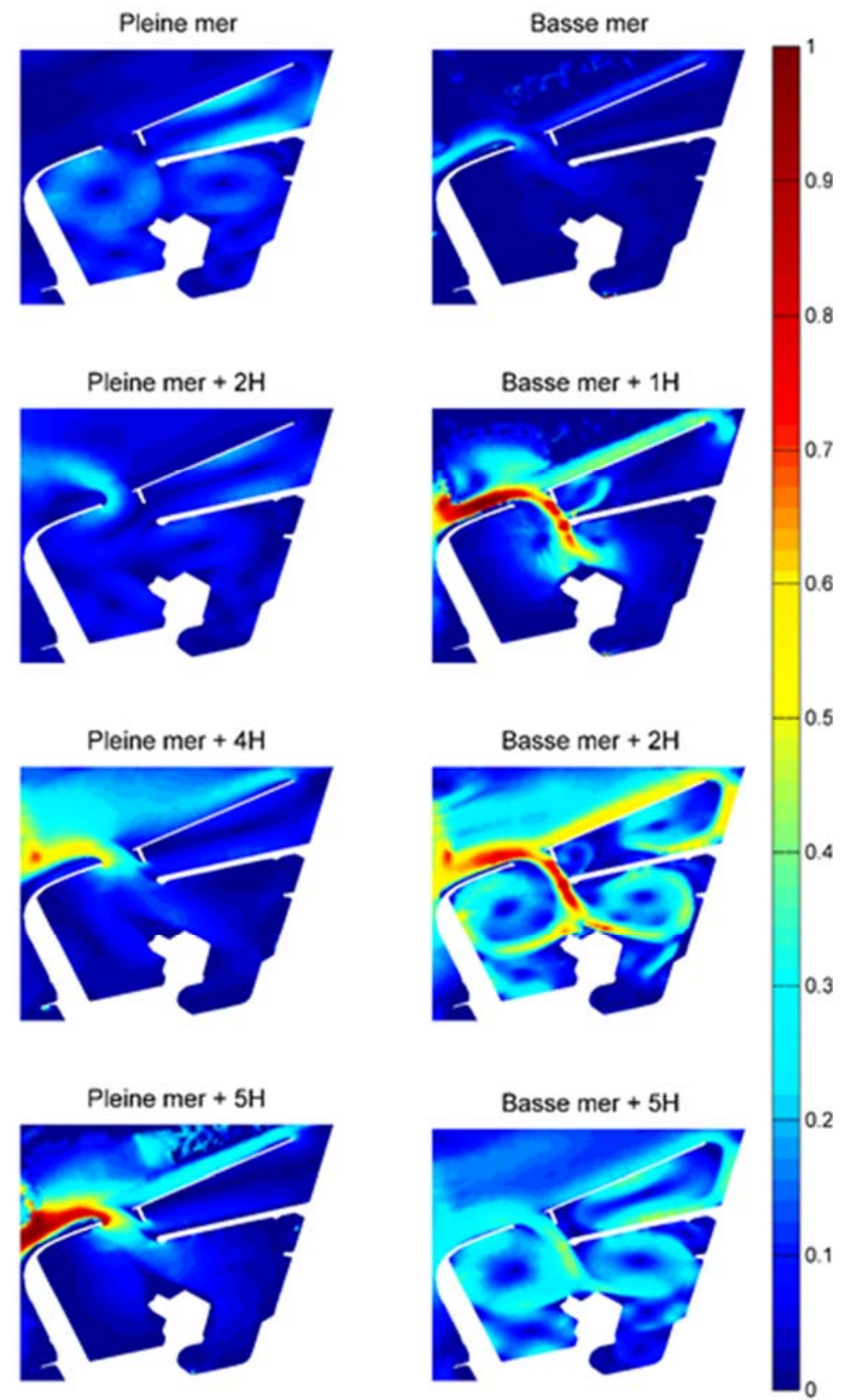

Figure 4. Courantologie, moyennée sur la verticale, du port des Minimes, à différents états de marée. Les résultats sont issus de simulations 3D pour une marée de vive-eaux (coefficient 110), sans effet de la météorologie. Les couleurs correspondent aux vitesses de courant, exprimées en $\mathrm{m} / \mathrm{s}$. 


\section{XVèmes Journées Nationales Génie Côtier - Génie Civil \\ La Rochelle, 29 au 31 mai 2018}

C'est d'ailleurs au chenal d'accès que l'on trouve les vitesses maximales qui approchent les $1,5 \mathrm{~m} / \mathrm{s}, 1 \mathrm{H}$ avant et après la basse mer. La situation est quasiment similaire en morte-eaux, avec des tourbillons moins définis, et des vitesses près de deux fois moins importantes que pour une marée de vive-eaux.

\subsection{Flux résiduels et influence de la météo}

Six cas ont été étudiés afin de comprendre quels étaient les flux résiduels sur l'ensemble du port, mais aussi au niveau de trois sections définies, visibles en figure 5. Le premier cas correspond à une situation avec marée seule; les 4 cas suivants correspondent à des situations avec marée et un vent théorique de 15 nœuds correspondant aux vents dominants dans la région (Ouest ; Nord-Ouest ; Nord-est ; Sud) ; le $6^{\text {ème }}$ cas correspond à une situation de tempête (vent de 30 nœuds d'origine Ouest). Tous ces cas d'études ont été simulés pour une marée de vive-eaux (VE) de coefficient 110 et une marée de morte-eaux (ME) de coefficient 35. Les résultats sont visibles sur le tableau 1 :

- Sans vent le gradient résiduel le plus important se fait via la section avec un départ des masses d'eaux hors du port des Minimes, compensé par un écoulement résiduel vers le bassin des Tamaris en section 3 et du bassin des Tamaris dans le bassin principal en section 2. En ME les flux résiduels sont plus faibles mais dirigés dans le même sens.

- Avec un vent d'ouest, les flux résiduels sont dans le même sens que sans vent, mais ils sont plus intenses notamment au niveau du flux entrant en section 3. Pour un vent de 30 nœuds, cette dynamique résiduelle est entretenue et 5 fois plus élevée.

- Le vent de nord-ouest a le même effet qu'un vent d'ouest sur les flux résiduels, avec une intensité moindre.

- Avec un vent de nord-est la circulation résiduelle est totalement remaniée. Les flux résiduels principaux se situent en section 3 avec un flux sortant important en ME. En VE l'effet du vent n'est pas suffisant pour éviter un flux résiduel sortant au niveau de la section 1 alors qu'en section 2 une gyre résiduelle est mise en place.

- Un vent de sud désorganise complètement la circulation tourbillonnaire au sein des différents bassins, que ce soit en VE ou ME. Alors que les flux résiduels sont dans le même sens que pour les simulations avec marée seule, il apparaît que le vent a une influence substantielle en ME avec un renversement du flux en section 1 et un quasiéquilibre en section 3 dû à la présence d'une gyre au peu active.

De manière générale les tourbillons résiduels à l'échelle des bassins sont maintenus en VE (excepté pour un vent constant de 30 nœuds ouest) alors qu'en ME ils sont totalement désorganisés. Toujours en VE, quel que soit le vent, le débit est dirigé vers l'extérieur en section 1 alors que le flux résiduel est beaucoup plus versatile en ME.

Enfin, le vent a pour effet, notamment en combinaison avec une ME, de créer de petites gyres locales au niveau des sections ce qui module le flux résiduel à l'échelle d'une marée et ce qui rajoute de la complexité. 


\section{Thème 1 - Hydrodynamique côtière}

Tableau 1. Flux résiduels de marée ( $\left.\mathrm{m}^{2} / \mathrm{s}\right)$ pour différents cas, en vive-eaux (VE) et en morte-eaux (ME) à travers les trois sections correspondant aux ouvertures principales du port des Minimes, visibles sur la figure 5. La couleur rouge correspond à nu flux sortant alors que la couleur bleue a été choisie pour un flux entrant dans un bassin à travers les sections définies.

\begin{tabular}{|c|c|c|c|c|c|c|}
\hline & \multicolumn{2}{|c|}{ Section } & \multicolumn{2}{|c|}{ Section } & \multicolumn{2}{|c|}{ Section } \\
\hline & $\mathbf{V}$ & $\mathbf{M}$ & $\mathbf{V}$ & $\mathbf{M}$ & $\mathbf{V}$ & M \\
\hline & $E$ & $E$ & $E$ & $\mathrm{E}$ & $\mathrm{E}$ & $E$ \\
\hline Sans & 34 & 12 & 21 & & & \\
\hline vent & 2 & $\beta$ & 1 & 58 & 29 & 7 \\
\hline & 76 & 10 & 53 & 74 & 86 & 12 \\
\hline $\mathbf{W}$ & $\beta$ & 58 & 5 & 5 & 1 & 24 \\
\hline & 17 & 22 & 13 & 16 & 21 & 27 \\
\hline W30 & 93 & 55 & p2 & 03 & 35 & 58 \\
\hline & 63 & 73 & 43 & 50 & 86 & 69 \\
\hline NW & p & 5 & 2 & $\beta$ & 5 & $\beta$ \\
\hline & & 10 & & 13 & & 16 \\
\hline NE & 74 & $\beta$ & 2 & t & 32 & 7 \\
\hline & 40 & 11 & 26 & 20 & 38 & \\
\hline $\mathbf{S}$ & 4 & 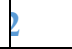 & & 7 & 7 & 0.4 \\
\hline
\end{tabular}

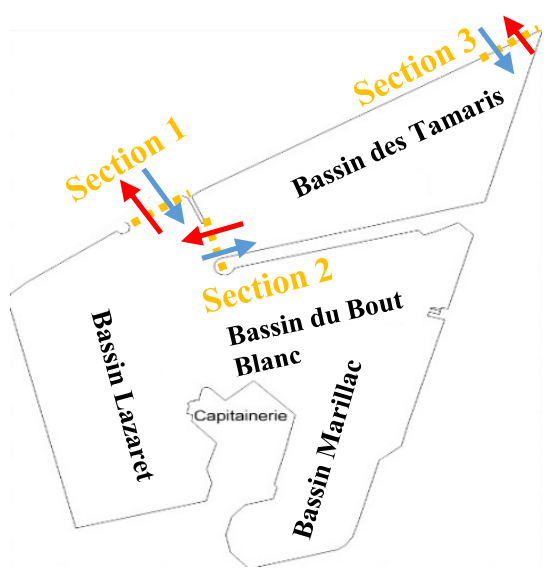

Figure 5. Sections correspondant aux ouvertures principales du port des Minimes.

\section{Discussion et conclusions}

Bien qu'il apparaisse que la marée soit le principal moteur des masses d'eau dans le port des Minimes, le vent semble avoir un impact non négligeable sur la circulation résiduelle dans ses différents bassins. Cependant, dans les résultats précédents nous nous sommes attachés à travailler seulement dans un environnement exempt de toute empreinte anthropique, sans pontons ni bateaux. Pourtant, sans considérer la circulation des bateaux, c'est plus du tiers de la surface totale du port des minimes qui est recouverte par des pontons et bateaux amarrés. Dans une étude préliminaire, les $15 \mathrm{~km}$ de pontons et plus de 4000 bateaux ont donc été ajoutés au modèle, de manière indirecte, via l'ajout d'un frottement en surface. Les simulations avec ces structures ont permis de constater qu'elles avaient un rôle non négligeable dans la circulation des masses d'eaux, notamment en réduisant drastiquement le régime tourbillonnaire dans 


\section{XVèmes Journées Nationales Génie Côtier - Génie Civil \\ La Rochelle, 29 au 31 mai 2018}

les bassins pendant le flot (figure 6). La circulation en jusant semble moins impactée, ce qui peut s'expliquer par le fait que les vitesses dans le chenal d'accès ne semblent pas être affectées par l'ajout des pontons. Cette étude est en cours de validation et apportera des informations sur les flux dans le port et donc sur les transferts sédimentaires et la remise en suspension suspension dans les bassins.
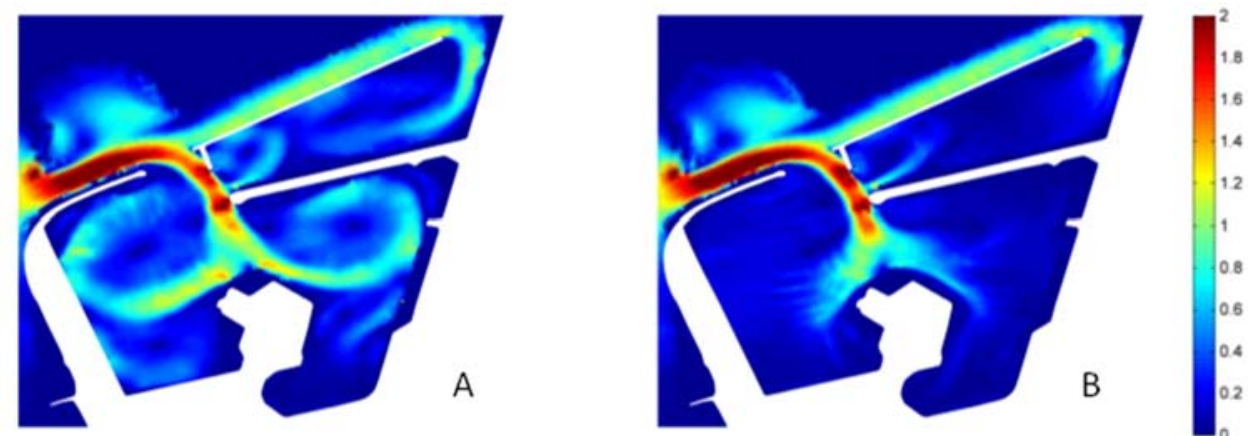

Figure 6. Comparaison de la courantologie, moyennée sur la verticale, du port des minimes au moment de marée "Basse mer $+2 H^{\prime}$. Les résultats sont issus de simulations

$3 D$, avec (B) et sans (A) pontons, pour une marée de vive-eaux (coefficient 110). Les couleurs correspondent aux vitesses de courant, exprimées en $\mathrm{m} / \mathrm{s}$.

\section{Références bibliographiques}

BERTIN X., CASTELlE B., CHAUMILLON E., BUTEL R., QUIQUE R. (2008). Longshore transport estimation and inter-annual variability of the longshore transport at a high-energy dissipative beach: the St Trojan beach, SW Oléron Island, France. Continental Shelf Research, Vol. 28, pp 1316-1332. https://doi.org/10.1016/j.csr.2008.03.005 BERTIN X., BRUNEAU N., BREILH J.F., FORTUNATO A.B., KARPYTCHEV M. (2012). Importance of wave age and resonance in storm surges: the case Xynthia, Bay of Biscay. Ocean Model, Vol. 42, pp 16-30. https://doi.org/10.1016/j.ocemod.2011.11.001

COLIN M. (1992). L'identité maritime des villes portuaires. Les Annales de la Recherche Urbaine.

POIRIER C., SAURIAU P.G., CHAUMILLON E., BERTIN, X. (2010). Influence of hydro-sedimentary factors on mollusc death assemblages in a temperate mixed tideand-wave dominated coastal environment: Implications for the fossil record. Continental Shelf Research, Vol. 30(17), pp 1876-1890. https://doi.org/10.1016/j.csr.2010.08.015 LE HIR P., KERVELLA S., STANISIERE J.-Y., ROBERT S. (2010). Modelling erosion, deposition and sediment fluxes in the Marennes-Oleron Bay: validation and discussion on uncertainties assessment. ISOBAY 12, XII International Symposium on Oceanography of the Bay of Biscay: Global changes in the Bay of Biscay. 3-6 May 2010, Brest, France. 
Thème 1 - Hydrodynamique côtière 